\title{
Bronchoscopic ablation of lung tumours: patient selection and technique
}

\author{
Ivan C. H. Siu ${ }^{1}$, Joyce W. Y. Chan ${ }^{1}$, Teofilo B. Manuel $\mathrm{II}^{1}$, Jenny C. L. Ngai ${ }^{2}$, Rainbow W. H. Lau ${ }^{1}$, \\ Calvin S. H. $\mathrm{Ng}^{1}$
}

${ }^{1}$ Division of Cardiothoracic Surgery, Department of Surgery, Prince of Wales Hospital, The Chinese University of Hong Kong, Hong Kong, China; ${ }^{2}$ Department of Medicine and Therapeutics, Prince of Wales Hospital, The Chinese University of Hong Kong, Hong Kong, China Contributions: (I) Conception and design: All authors; (II) Administrative support: CSH Ng, RWH Lau, JWY Chan; (III) Provision of study materials or patients: CSH Ng, RWH Lau, JWY Chan; (IV) Collection and assembly of data: All authors; (V) Data analysis and interpretation: All authors; (VI) Manuscript writing: All authors; (VII) Final approval of manuscript: All authors.

Correspondence to: Dr. Calvin S. H. Ng, MD, FRCS. Professor, Division of Cardiothoracic Surgery, Department of Surgery, The Chinese University of Hong Kong, Prince of Wales Hospital, Hong Kong, China. Email: calvinng@surgery.cuhk.edu.hk.

\begin{abstract}
With increasing numbers of lung nodules and ground glass opacities (GGO) being identified through increased computed tomography (CT) screening, thoracic surgeons nowadays are faced with an increasing demand for treatment for suspicious nodules. While surgical lobectomy remains the gold standard, for surgically unfit patients, this may not always be possible. Many developments have been made in alternative therapies including stereotactic body radiation therapy (SBRT), as well as various percutaneous ablation techniques, specifically with the use of radiofrequency ablation (RFA) and microwave ablation (MWA). This, combined with the development of advanced localisation techniques for lung nodules and GGO in the advent of hybrid operating theatres such as electromagnetic navigation bronchoscopy (ENB), have paved the way for bronchoscopic intervention as becoming the new frontier. Our institution has taken the advantage of these developments and have embarked on the journey of developing an "incision-less" bronchoscopic ablative therapy utilising ENB and MWA to patients with suspicious lung lesions. We have successfully demonstrated safety and feasibility with this technique, but through our experience, we have identified specific areas of consideration both preoperatively and intraoperatively. In this article we describe our preoperative and operative experience for ENB guided MWA, with particular emphasis on patient selection and technical pearls.
\end{abstract}

Keywords: Microwave ablation (MWA); electromagnetic navigation bronchoscopy (ENB); transbronchial ablation; hybrid operating room; lung cancer

Received: 22 July 2021; Accepted: 11 October 2021; Published: 20 October 2022.

doi: 10.21037 /jovs-21-45

View this article at: https://dx.doi.org/10.21037/jovs-21-45

\section{Introduction}

While surgical lobectomy remains the gold standard treatment for patients with early stage non-small cell lung cancer, various factors may preclude patients from surgery, and thus a wide array of alternative treatment modalities have become available over the years. In addition to sublobar resection $(1,2)$ and stereotactic body radiation therapy (SBRT) (3), image guided local ablative therapies using radiofrequency ablation (RFA), microwave ablation (MWA) and cryoablation via a percutaneous route have demonstrated feasibility in select patient groups $(4,5)$. With the increasing use of low dose computed tomography (CT) scans for screening (6), thoracic surgeons are faced with an ever growing number of small lung nodules and ground glass opacities (GGO) of indeterminate nature, with potential for malignant change (7). This has paved the way for the development of localization techniques 
such as CT guided hookwire or microcoil placement, virtual bronchoscopy and electromagnetic navigation bronchoscopy (ENB), to tackle the challenge of intraoperative identification of these small lesions for diagnostic and therapeutic purposes $(8,9)$. The rising popularity of the hybrid operating theatre has also allowed for the marriage of such techniques with minimally invasive surgery to provide an efficient operating environment for localisation and therapy in one single setting (9-13).

The amalgamation of these developments have seen the rise of bronchoscopic intervention as a minimally invasive diagnostic and therapeutic option. Using bronchoscopy guided localization followed by transbronchial RFA ablation, a Japanese group was able to demonstrate feasibility and safety with this novel technique (14). Compared with percutaneous ablation, advantages of bronchoscopic therapy include an overall reduction in complication rates, with reduced risk of pleural-based complications including pneumothorax, pleural effusion, bronchopleural fistula, and needle tract seeding $(14,15)$. The theoretical advantages of MWA compared with RFA include; less limitation by the high impedance of lung tissue, less heat sink effect, larger and more reproducible ablation zones, higher intratumoral temperatures as well as faster ablation times $(5,15,16)$. With these in mind, our institution is one of the first to utilise MWA in the hybrid operating theatre using ENB guidance for malignant or suspicious lung nodules (17), and the following article will describe our experience, starting from patient selection to intra-operative techniques.

\section{Patient selection}

Between the period from March 2019 to May 2021, a total of 68 patients had undergone ENB guided MWA at our institution. All patients had a plain fine cut CT within 3 months from the scheduled date of ablation for preoperative planning purposes. Patients selected all had biopsy proven malignant, or radiologically suspicious lung nodules who were either not surgical candidates due to comorbidities/high surgical risk or have declined surgery. Despite this, all candidates would still have to tolerate single lumen endotracheal tube intubation for the procedure, under general anaesthesia. Patients suffering from Global Initiative for Chronic Obstructive Lung Disease (GOLD) stage IV emphysema were excluded in view of higher general anaesthesia risk and procedural risks, as were patients who were pregnant. In the future, with more safety data and experience, patients with chronic obstructive pulmonary disease (COPD) IV can be included as well as it stands as one of the major risk factors for malignant incidental malignant nodules. For those patients with biopsy proven lung cancer, distant metastases need to be excluded with CT or positron emission tomography (PET)/ CT while suspicious nodal involvement had to be ruled out with histology, so that eligible nodules for bronchoscopic MWA are effectively T1N0M0. For patients with lung metastases, ablation was only offered if the primary tumour was treated with curative intent and other oligometastases were treatable. For patients with certain metallic implants, for example sternal wires in patients with previous cardiac surgery, the accuracy of ENB for localisation of the target lesion is not affected (18), and bronchoscopic MWA would still be feasible. Patients with cardiac pacemakers however, would be contraindicated for the procedure based on manufacturer's current recommendation. Concurrent use of anticoagulation such as warfarin or direct oral anticoagulants like apixaban, is not a contraindication to bronchoscopic MWA, but would require preoperative cessation. In our experience, for patients on apixaban, we stop the drug 2 days before the procedure, and if no complications are encountered, the anticoagulant can be restarted on postoperative day 1. Depending on the indication of anticoagulation, peri-operative bridging therapy with intravenous heparin may be required; the infusion is stopped 6 hours before the ablation procedure, and is restarted after the procedure until the usual anticoagulant is given.

There are also particular considerations for lesion size, shape and location. Lesions should have a maximal diameter of less than $30 \mathrm{~mm}$, and at least $5 \mathrm{~mm}$ away from major blood vessels ( $>3 \mathrm{~mm}$ in diameter). The target lesion should also not be abutting main stem bronchus or mediastinal structures to avoid thermal injury to important visceral structures. Thus, lesions most suitable for ablation would be located in the middle and outer thirds of the lung. Special consideration should be taken for lesions with ablation zones that may cause thermal injury to phrenic nerve and brachial plexus. The presence of a "bronchus sign" whereby a bronchus can be seen leading directly to the lesion is favourable, for ease of navigation during the procedure (Figure 1). The absence of a nearby bronchus however, does not preclude feasibility of the procedure, but would require additional manoeuvring during navigation or transbronchial access tools. Shape of the lesions are also important to determine the subsequent MWA; irregularly shaped i.e., longitudinal or "dumb-bell" shaped lesions, may require additional ablation via various techniques, due to inadequate 


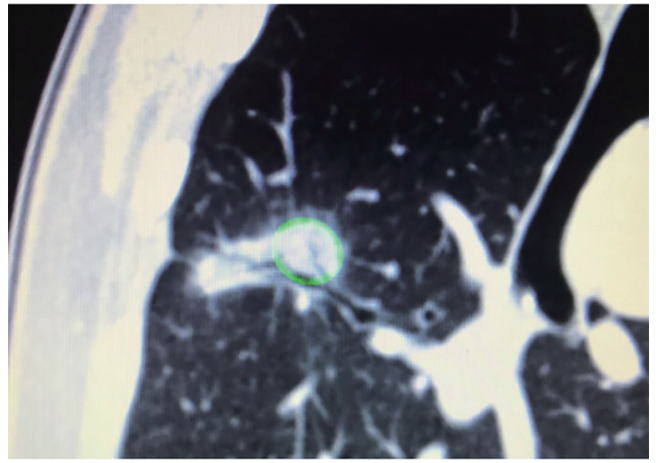

Figure 1 The "bronchus sign" seen in this particular lesion, whereby an airway is seen leading directly into the lesion.
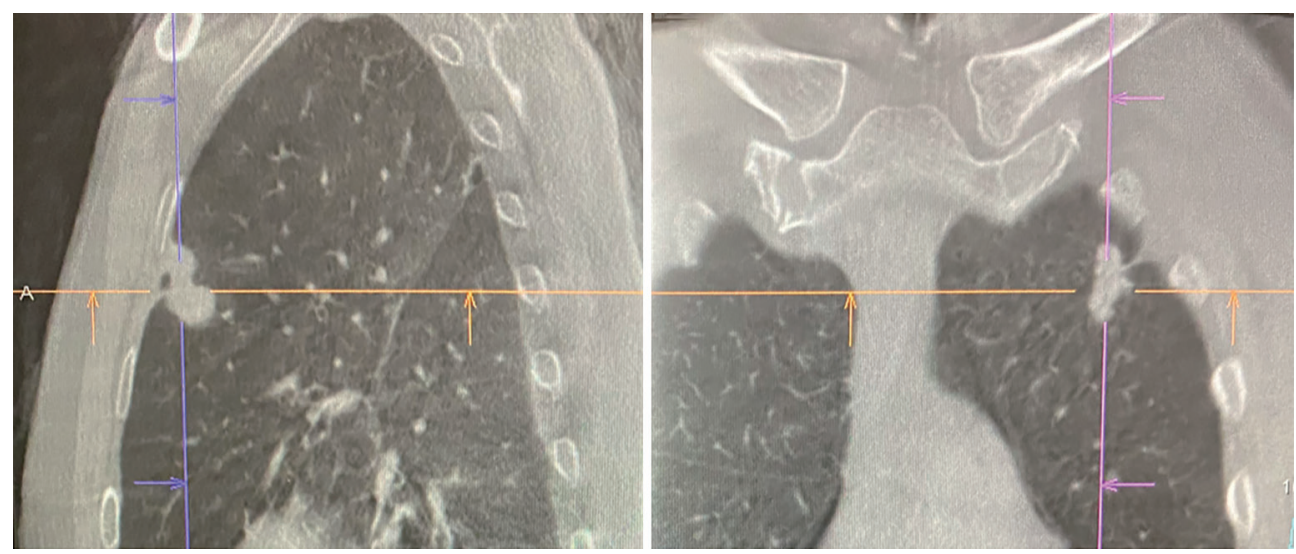

Figure 2 This particular lesion is shaped longitudinally, and has a "dumbbell" appearance, which poses as a challenge for subsequent ablation to ensure adequate coverage of the ablation zone.

single ablation zone coverage (Figure 2) (17).

\section{Technique}

The usual setup in our hybrid operating theatre includes the Artis Zeego Multi-axis Robotic Imaging System (Siemens Healthcare AG, Forchheim, Germany) with cone beam CT (CBCT) and fluoroscopic capabilities with $\mathrm{PURE}^{\circledR}$ platform software for segmentation and overlay (Figure 3). The patient is put under general anaesthesia with single lumen endotracheal intubation, lying supine. The caliber of the endotracheal tube needs to be large, preferably size 8 , to allow good collateral airflow around the large bronchoscope during a long procedure to avoid atelectasis and carbon



Figure 3 Usual setup of the hybrid operating theatre for ENB guided MWA, with the C-arm of the CBCT seen, as well as fluoroscopy shown on screen. ENB, electromagnetic navigation bronchoscopy; MWA, microwave ablation; CBCT, cone beam computed tomography. 


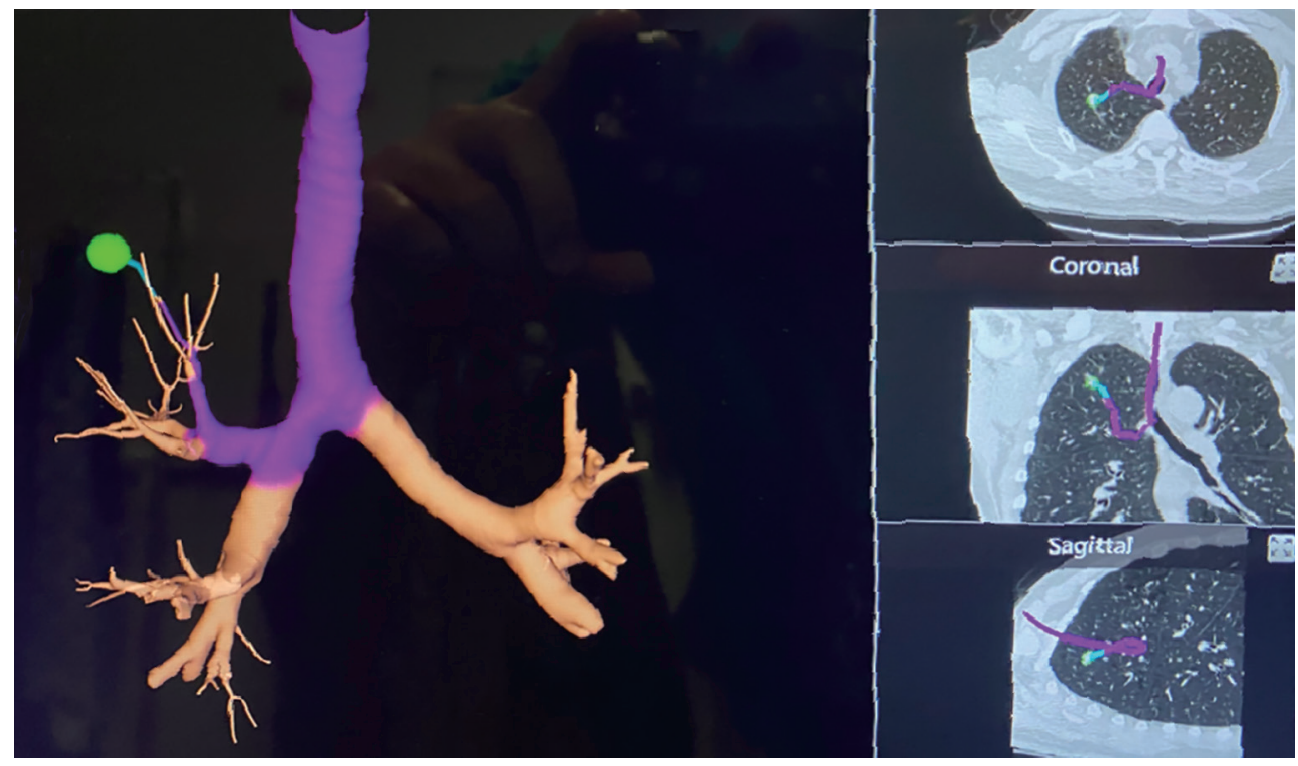

Figure 4 After registration of the target lesion and surrounding airways, a virtual route would be created for navigation.
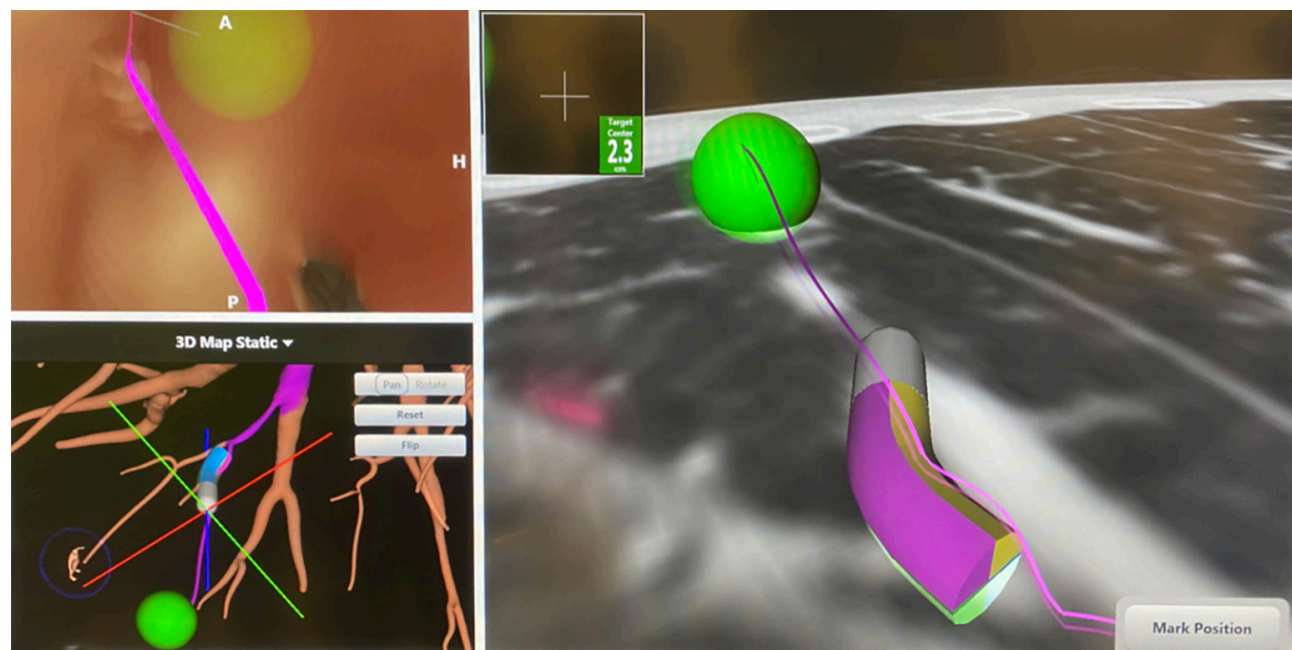

Figure 5 The virtual bronchoscopic route as seen during navigation towards the target lesion, represented by the green ball.

bronchoscopic route (Figure 5). The choice of EWC angulation to be used for navigation is important bearing in mind the airway anatomy and the relative position of the lesion to its access airway. Our usual choice would be either EDGE ${ }^{\mathrm{TM}}$ 180-degree EWC or the Medial Firm catheter. Fluoroscopic guidance is also employed to ascertain positioning of catheter tip in relation to the target lesion in real time (Figure 6), where the target lesion is marked on the fluoroscopy screen using iGuide (Siemens Healthineers, Germany). Once satisfactory position of catheter tip is thought to be reached based on ENB virtual bronchoscopy and/or fluoroscopy, the LG is exchanged to a needle, and another CBCT is performed to confirm position, to guide further adjustment if deemed necessary. To facilitate the rotation of the C-arm of the CBCT, while at the same time ensuring stability of the bronchoscope and the catheter within the working channel during the scan, it is our practice to secure the entire apparatus on a foam lined box at the head side of patient (Figure 7). This allows for CBCT without the need for the operator to hold the 


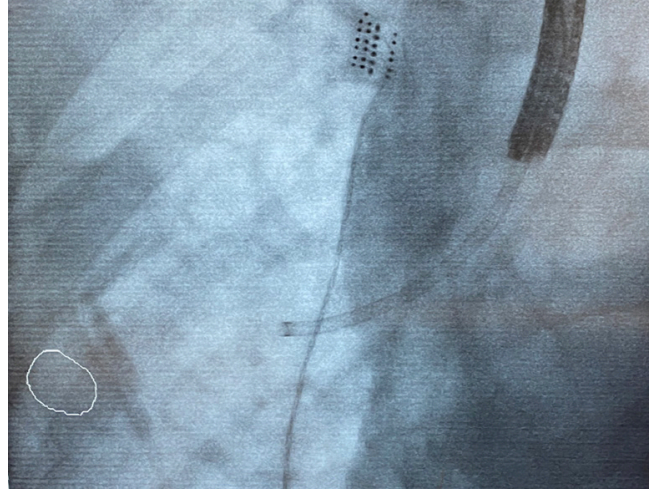

Figure 6 Fluoroscopy showing an irregularly shaped lesion marked with white tracings. The ablation catheter extends from within the EWC of the bronchoscope, and its tip is positioned inside lesion. It is often necessary to obtain fluoroscopy at two different angles to confirm proximity of catheter tip to the lesion in a threedimensional space. EWC, extended working channel.

apparatus. Some bronchoscope stabilization systems are also now commercially available which would, likewise provide security to the entire apparatus without the operator.

In lesions without a favourable "bronchus sign", additional transbronchial access may be required to successfully reach the lesion for ablation, using the CrossCountry ${ }^{\mathrm{TM}}$ Transbronchial Access Tool (Covidien ${ }^{\mathrm{TM}}$, Plymouth, MN, USA). A needle is first used to puncture the bronchus, followed by tract dilatation to create a tunnel for subsequent transbronchial access. The presence of blood vessels along the projected transbronchial access path should be noted during pathway planning and should be avoided.

Once the desired location has been reached, the needle is removed from the EWC and exchanged with the Emprint ${ }^{\mathrm{TM}}$ Ablation Catheter with Thermosphere ${ }^{\mathrm{TM}}$ technology (Covidien ${ }^{\mathrm{TM}}$, Plymouth, MN, USA), and the tip of the MWA catheter is unsheathed by retracting the EWC. Another CBCT is performed to confirm the location of the catheter with respect to the target lesion, in order to plan the predicted ablation zone using the PURE ${ }^{\circledR}$ platform (Siemens Heathineers, Germany) software, with adequate margin, defined as a minimum of $5 \mathrm{~mm}$ (Figure 8). Minor adjustments of the ablation catheter can be made under fluoroscopy to optimize the ablation margin for the lesion, but will subsequently require another CBCT prior to initiating ablation. The size of ablation zone varies with the amount of power chosen and the duration of ablation. Fluoroscopy during the ablation is also carried out at regular

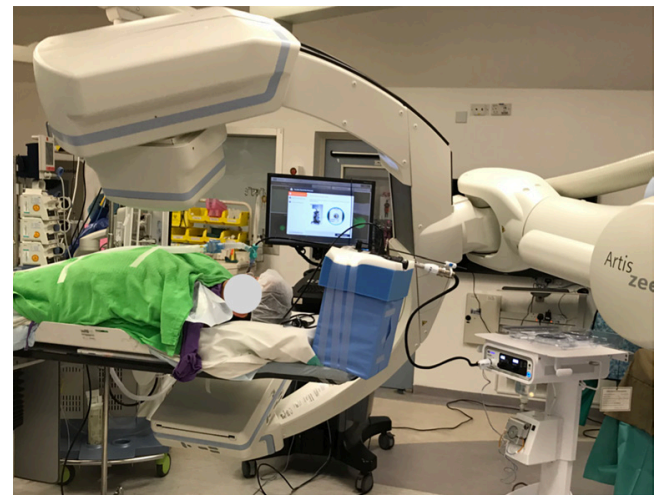

Figure 7 The bronchoscope, EWC and ablation catheter is seen supported by the blue box, lined by foam and secured with tape around the operating table. The supporting foam box is placed at the head side of patient to avoid collision by $\mathrm{C}$-arm during rotation. EWC, extended working channel.

time intervals, usually every 3 minutes, to ensure stable position of the catheter and to look for any inadvertent dislodgement. A cooling period of 10 minutes post-ablation is required, prior to another $\mathrm{CBCT}$ with the ablation catheter left in situ to evaluate post-ablation zone coverage and margins. If the lesion is not well seen after ablation, pre- and post-ablation CBCT are overlayed to determine the actual margin achieved. If the post-ablation scan demonstrates success, then the procedure is completed with removal of the catheter, EWC, followed by bronchial toileting and subsequent extubation.

\section{Challenges}

In nodules which are close to fissure or pleura, further consideration needs to be taken during navigation or ablation. Although there is an advantage with bronchoscopic ablation compared with percutaneous ablation in reducing risk of pneumothorax (15), it is still a potential concern in lesions that lie close to the pleura. In our experience however, as long as inadvertent pleural puncture (Figure 9) is avoided during navigation with the LG or deployment of the ablation catheter, even if the ablation zone reaches the pleura, the risk of pneumothorax is low. In fact, the ablation zone reached pleura in $66.7 \%$ of our cases, yet the pneumothorax rate is only $8.2 \%$. In order to reduce the risk of inadvertent pleura puncture, it is our practice to utilise fluoroscopy for real time imaging during deployment of needles or catheters, and the 

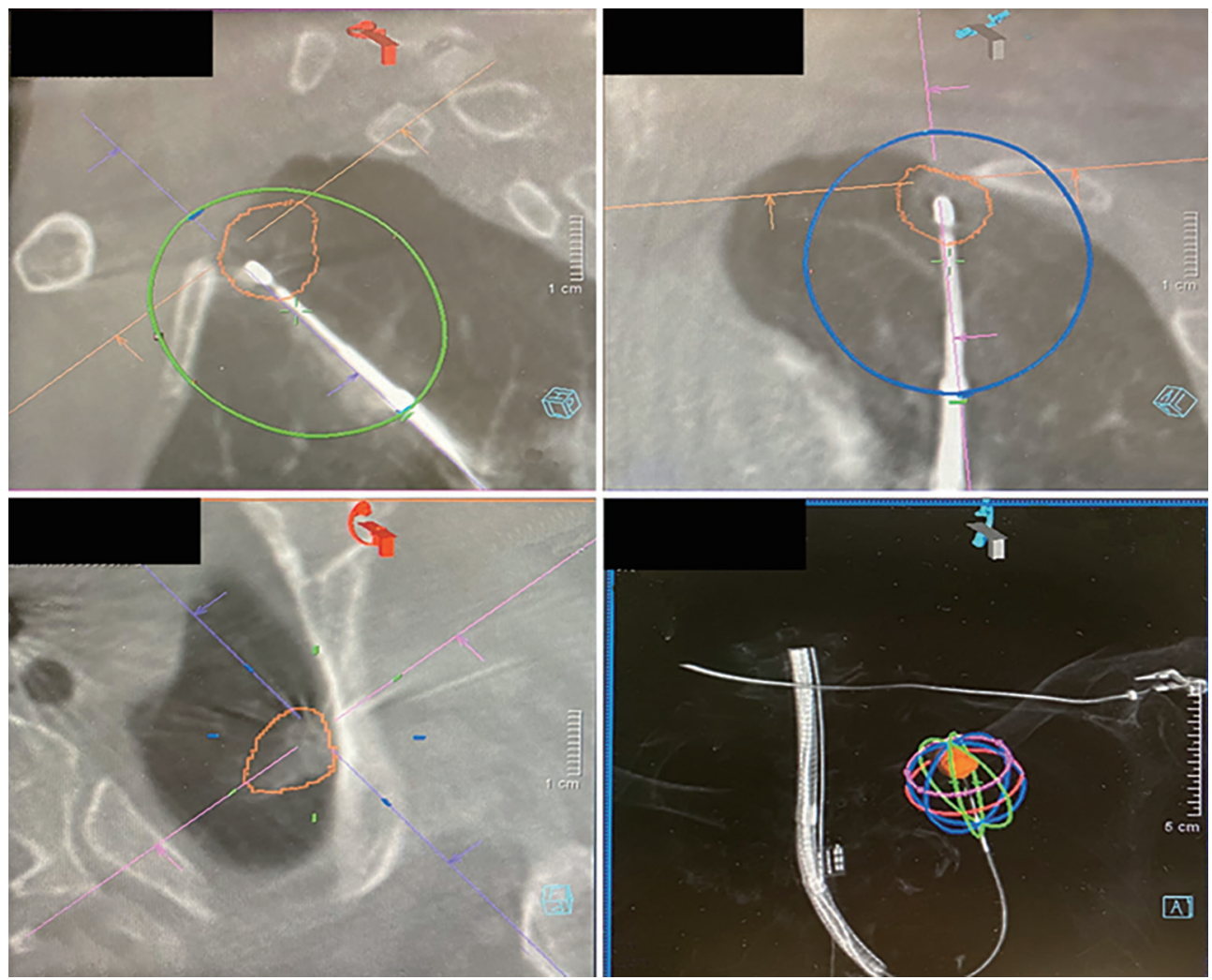

Figure 8 The target lesion is punctured by the ablation catheter on three axes CBCT image. The blue and green ovoids are the predicted ablation zones while the orange marking represents the lesion. The right lower image shows the combined predicted ablation zone, represented by the "cage" surrounding the lesion. CBCT, cone beam computed tomography.

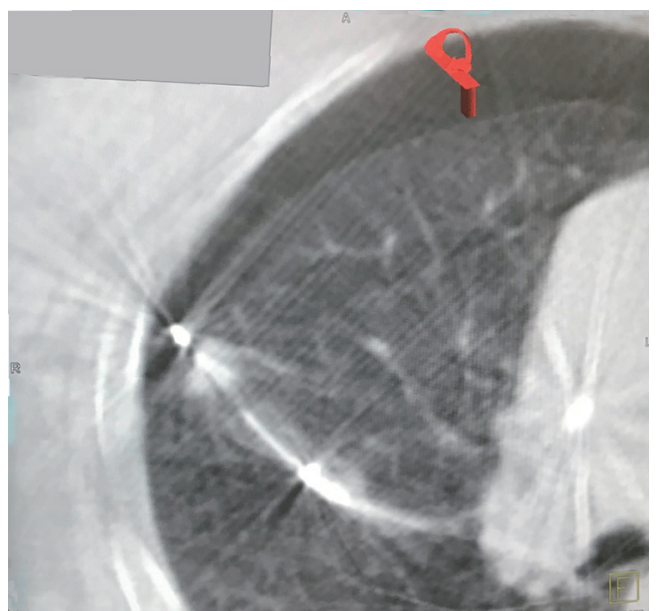

Figure 9 The ablation catheter was advanced more than required leading to pleural puncture, resulting in pneumothorax. use of iGuide (Siemens Healthineers, Germany) overlay to mark the edge of the pleura/fissure on the fluoroscopy screen, is useful to allow the operator to visualise the lung margin (Figure 10). In the unfortunate event that a pneumothorax does occur during the procedure, it is imperative that this is recognised early and a chest drain inserted immediately to allow full re-expansion of the lung. This is followed by confirmation of the resolution of the pneumothorax with a CBCT scan, and the ablation procedure is continued with the chest drain in situ.

Sometimes a single ablation zone does not provide adequate coverage of the entire lesion with appropriate margins, thus requiring a second ablation. This can sometimes be a planned event, especially if the lesion is sizeable or oddly shaped as previously mentioned (Figure 2), or it can be unplanned and noted only after the post- 


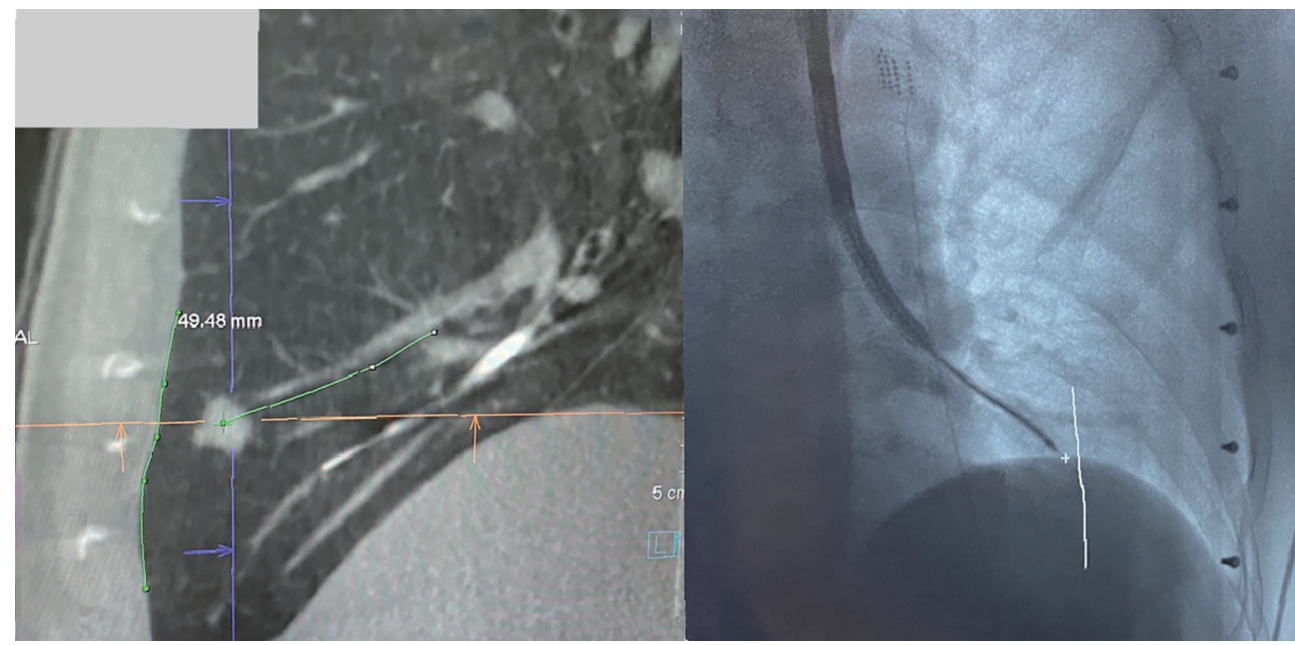

Figure 10 Seen on the CT image, the green lines have marked both the airway towards the target lesion, as well as the edge of the pleura. This is then overlay onto the fluoroscopy screen (represented by the white line) to allow the operator to know the limit during deployment of needle or catheter. CT, computed tomography.

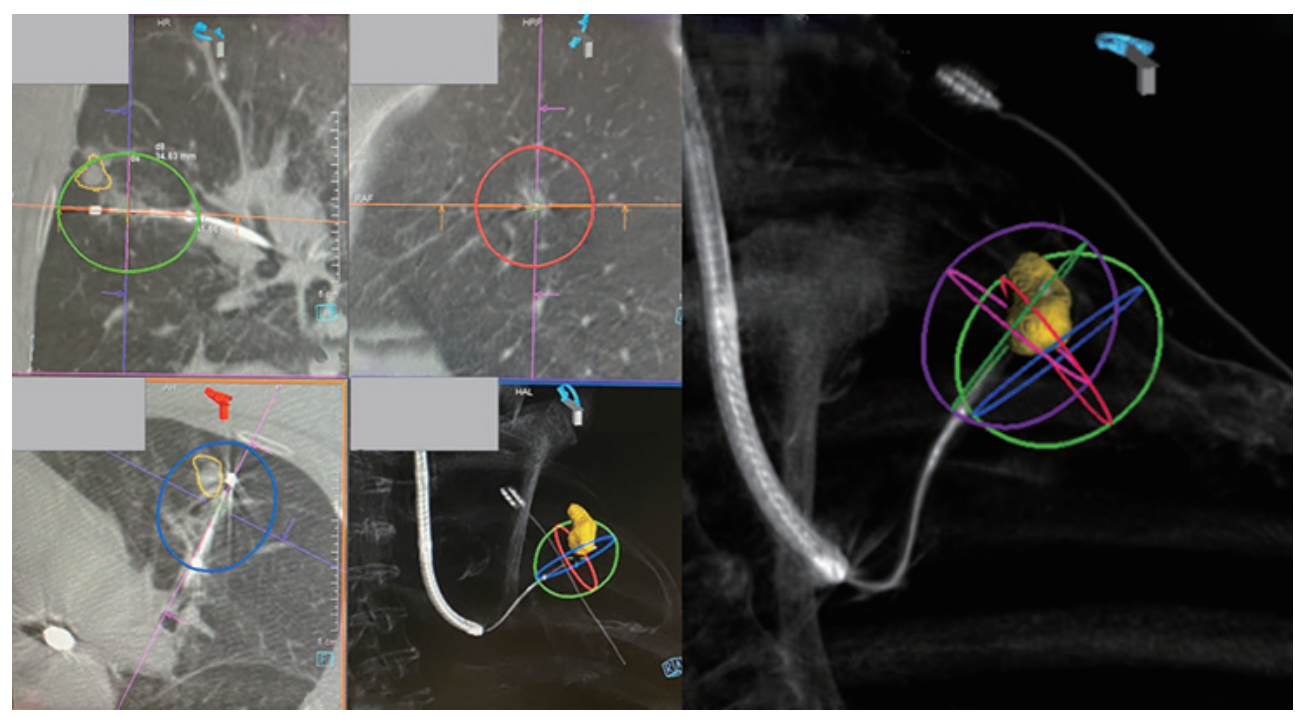

Figure 11 A relatively large and oddly shaped nodule is shown. The first predicted ablation zone (green, red, blue rings) as depicted on the four left side panels was inadequate to cover the entire lesion. Hence, a "bracket" double ablation was performed as seen on the right side panel. The catheter was re-navigated to a nearby position under ENB, and an additional ablation zone (purple ring) was created, with the combined ablation zone covering the target lesion with adequate margin. ENB, electromagnetic navigation bronchoscopy.

ablation CT revealing a smaller than expected ablation zone. Double ablation can be achieved by a repeat ablation at the same position, or pulling back the catheter and re-ablating. Sometimes a "bracket ablation" will be required, which requires re-navigation using $\mathrm{ENB}$ to a new desired location and then re-ablating due to the limitation in exact positioning of the ablation catheter to achieve an adequate margin with a single ablation zone in large lesions (Figure 11). Irrespective of the reason for or the type of double ablation performed, following the first ablation, interpretation of the lesion boundaries and ablation margins can be challenging. Double ablations tend to be for larger lesions, and higher vigilance 
for recurrence is needed in these ablated lesions during postablation serial imaging.

In cases where histological proof of malignancy is not obtained preoperatively, a concomitant ENB guided biopsy can also be performed just prior to the deployment of the MWA catheter. This however can potentially complicate the subsequent ablation procedure, as local haemorrhage following biopsy is sometimes encountered, and this would obscure the target lesion as seen on subsequent CT scans. This also makes the final assessment of the adequacy of the ablation zone difficult, and thus preoperative biopsy should be considered instead. If the lesion is small and difficulty with biopsy is anticipated, but the lesion is radiologically and clinically suspicious, ablation without biopsy can be considered.

\section{Conclusions}

ENB guided MWA is the culmination of advancing medical technology, and one of the many growing developments in the armamentarium in the field of thoracic surgery. Our experience with ENB guided MWA of lung lesions have opened up an alternative method for patients who would otherwise not be able to receive surgical treatment. The advantages of a completely incision-less treatment are reflected by a shorter overall inpatient hospital stay, reduced postoperative pain, reduction in pleural-based complications, and ultimately stands as a safe and feasible treatment (17).

\section{Acknowledgments}

Funding: This work was supported by University Grants Committee (UGC) Research Grants, Council (RGC), Hong Kong, Grant No. 14119019.

\section{Footnote}

Provenance and Peer Review: This article was commissioned by the Guest Editors (Hiran Fernando and John Lazar) for the series "Innovations in Robotic VATS and Bronchochoscopic Procedures" published in Fournal of Visualized Surgery. The article has undergone external peer review.

Peer Review File: Available at https://jovs.amegroups.com/ article/view/10.21037/jovs-21-45/prf

Conflicts of Interest: All authors have completed the
ICMJE uniform disclosure form (available at https:// jovs.amegroups.com/article/view/10.21037/jovs-2145/coif). The series "Innovations in Robotic VATS and Bronchochoscopic Procedures" was commissioned by the editorial office without any funding or sponsorship. CSHN is a consultant for Johnson and Johnson; Medtronic, USA; and Siemens Healthineers. CSHN serves as an unpaid editorial board member of fournal of Visualized Surgery from June 2021 to May 2023. The authors have no other conflicts of interest to declare.

Ethical Statement: The authors are accountable for all aspects of the work in ensuring that questions related to the accuracy or integrity of any part of the work are appropriately investigated and resolved.

Open Access Statement: This is an Open Access article distributed in accordance with the Creative Commons Attribution-NonCommercial-NoDerivs 4.0 International License (CC BY-NC-ND 4.0), which permits the noncommercial replication and distribution of the article with the strict proviso that no changes or edits are made and the original work is properly cited (including links to both the formal publication through the relevant DOI and the license). See: https://creativecommons.org/licenses/by-nc-nd/4.0/.

\section{References}

1. Zhao ZR, Situ DR, Lau RWH, et al. Comparison of segmentectomy and lobectomy in stage IA adenocarcinomas. J Thorac Oncol 2017;12:890-6.

2. Rami-Porta R, Tsuboi M. Sublobar resection for lung cancer. Eur Respir J 2009;33:426-35.

3. Shinde A, Li R, Kim J, et al. Stereotactic body radiation therapy (SBRT) for early-stage lung cancer in the elderly. Semin Oncol 2018;45:210-9.

4. Zhao ZR, Lau RWH, Ng CSH. Catheter-based alternative treatment for early-stage lung cancer with a high-risk for morbidity. J Thorac Dis 2018;10:S1864-70.

5. Alexander ES, Dupuy DE. Lung cancer ablation: technologies and techniques. Semin Intervent Radiol 2013;30:141-50.

6. National Lung Screening Trial Research Team; Aberle DR, Adams AM, et al. Reduced lung-cancer mortality with low-dose computed tomographic screening. N Engl J Med 2011;365:395-409.

7. Yu PSY, Chan JWY, Lau RWH, et al. Screening-detected pure ground-glass opacities: malignant potential beyond 
conventional belief? Transl Lung Cancer Res 2020;9:816-8.

8. Chan JWY, Lau RWH, Ng CSH. Electromagnetic navigation bronchoscopy fiducial marker margin identification plus triple dye for complete lung nodule resection. JTCVS Tech 2020;3:329-33.

9. Zhao ZR, Lau RW, Ng CS. Hybrid theatre and alternative localization techniques in conventional and singleport video-assisted thoracoscopic surgery. J Thorac Dis 2016;8:S319-27.

10. Zhao ZR, Lau RWH, Ng CSH. Hybrid theater and uniportal video-assisted thoracic surgery: the perfect match for lung nodule localization. Thorac Surg Clin 2017;27:347-55

11. Awais O, Reidy MR, Mehta K, et al. Electromagnetic navigation bronchoscopy-guided dye marking for thoracoscopic resection of pulmonary nodules. Ann Thorac Surg 2016;102:223-9.

12. Yu PSY, Chu CM, Lau RWH, et al. Hybrid theater facilitates lung-preserving multimodal treatment for multiple pulmonary metastases. Ann Thorac Surg 2021;111:e89-92.

13. Chan JWY, Yu PSY, Lau RWH, et al. Hybrid operating room-one stop for diagnosis, staging and treatment of

doi: $10.21037 /$ jovs-21-45

Cite this article as: Siu ICH, Chan JWY, Manuel TB 2nd, Ngai JCL, Lau RWH, Ng CSH. Bronchoscopic ablation of lung tumours: patient selection and technique. J Vis Surg 2022;8:36. early stage NSCLC. J Thorac Dis 2020;12:123-31.

14. Koizumi T, Tsushima K, Tanabe T, et al. Bronchoscopyguided cooled radiofrequency ablation as a novel intervention therapy for peripheral lung cancer. Respiration 2015;90:47-55.

15. Pritchett MA, Schirmer CC, Laeseke P. Melting the tip of the iceberg: bronchoscopic-guided transbronchial microwave ablation. Transl Lung Cancer Res 2020;9:960-3.

16. Brace CL, Hinshaw JL, Laeseke PF, et al. Pulmonary thermal ablation: comparison of radiofrequency and microwave devices by using gross pathologic and CT findings in a swine model. Radiology 2009;251:705-11.

17. Chan JWY, Lau RWH, Ngai JCL, et al. Transbronchial microwave ablation of lung nodules with electromagnetic navigation bronchoscopy guidance-a novel technique and initial experience with 30 cases. Transl Lung Cancer Res 2021;10:1608-22.

18. Fernandez RAS, Yu PSY, Chan JWY, et al. The comparison of electromagnetic with virtual bronchoscopic navigation accuracy at hybrid operating room in patient with sternal wires. AME Case Rep 2020;4:5. 